\title{
Cross-calibration of Probabilistic Forecasts
}

\section{- Supplementary Material- Further Examples and Score Regression Approach}

\author{
Christof Strähl and Johanna Ziegel \\ Institute of Mathematical Statistics and Actuarial Sciences, \\ University of Bern, Switzerland \\ e-mail: christof.straehl@stat.unibe.ch; johanna.ziegel@stat.unibe.ch
}

\section{Contents}

1 The Cross-calibration Test of Feinberg and Stewart (2008) 2

2 Diagnostic plots for Assessing Cross-calibration . . . . . . 3

3 The Score Regression Approach . . . . . . . . . . . . . . 4

4 Testing for Marginal Cross-calibration . . . . . . . . . . 9

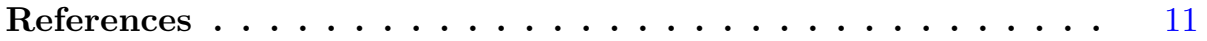




\section{The Cross-calibration Test of Feinberg and Stewart (2008)}

The cross-calibration test suggested by Feinberg and Stewart (2008) uses the following framework, which we review here only in the case of two forecasters for simplicity. Let $\Omega=\left\{\left(\omega_{t}\right)_{t=0,1, \ldots} \mid \omega_{t} \in\{0,1\}\right\}$ denote the space of all possible realizations and let $n>4$ be an integer. Divide the interval $[0,1]$ into $n$ equal closed subintervals $[0,1 / n], \ldots,[(n-1) / n, 1]$. At time $t$, forecaster $j, j=1,2$, makes a prediction which is given as an interval $I_{t}^{j} \in\{[0,1 / n], \ldots,[(n-1) / n, 1]\}$. It gives bounds on the predictive probability that the next realization $\omega_{t}$ is equal to one. The cross-calibration test is defined over the sequence of forecastobservation triples $\left(I_{t}^{1}, I_{t}^{2}, \omega_{t}\right)_{t=0}^{\infty}$. For any pair $\ell=\left(\ell_{1}, \ell_{2}\right) \in\{1, \ldots, n\}^{2}$ and any time $T$, let

$$
\nu_{T}^{\ell}=\sum_{t=0}^{T} \mathbb{1}\left(I_{t}^{1}=\left[\frac{\ell_{1}-1}{n}, \frac{\ell_{1}}{n}\right], I_{t}^{2}=\left[\frac{\ell_{2}-1}{n}, \frac{\ell_{2}}{n}\right]\right),
$$

which is the number of times up to $T$, that the forecasting profile $\ell$ is chosen. For $\nu_{T}^{\ell}>0$, the frequency of realizations equal to one conditional on the forecasting profile is given by

$$
f_{T}^{\ell}=\frac{1}{\nu_{T}^{l}} \sum_{t=0}^{T} \omega_{t} \mathbb{1}\left(I_{t}^{1}=\left[\frac{\ell_{1}-1}{n}, \frac{\ell_{1}}{n}\right], I_{t}^{2}=\left[\frac{\ell_{2}-1}{n}, \frac{\ell_{2}}{n}\right]\right) .
$$

Forecaster $j$ passes the cross-calibration test at the outcome $\left(I_{t}^{1}, I_{t}^{2}, \omega_{t}\right)_{t=0}^{\infty}$ if

$$
\limsup _{T \rightarrow \infty}\left|f_{T}^{\ell}-\frac{2 \ell_{j}-1}{2 n}\right| \leq \frac{1}{2 n}
$$

for every $\ell$ satisfying $\lim _{T \rightarrow \infty} \nu_{T}^{\ell}=\infty$.

It is shown in Feinberg and Stewart (2008) that a forecaster who is aware of the distribution of $\left(\omega_{t}\right)_{t=0}^{\infty}$ passes the cross-calibration test with probability one, no matter which strategy the other forecaster uses. From a theoretical point of view, this is an interesting result. However, testing empirically if a forecast is cross-calibrated is rather difficult. The problem is that already if $n=5$, there are 25 forecasting profiles to consider. For each of these profiles the empirical frequency conditioned on that profile should lie inside the predicted interval of the cross-calibrated forecaster. But some profiles are hardly ever predicted and therefore the number of observations $T$ needs to be very large. We illustrate this problem with a simple simulation example. It has been implemented in $\mathrm{R}(\mathrm{R}$ Core Team, 2015) like all further simulations in this supplement.

Example 1.1. In this example we consider the setting of the cross-calibration test of Feinberg and Stewart (2008) described in Section 1 of the paper. Let $B_{t}$, $C_{t}, t=0, \ldots, T$ be independent beta random variables with parameters $(3,5)$ for $B_{t}$ and $(2,1.5)$ for $C_{t}$. We simulate a (finite) stochastic process $\left(\omega_{t}\right)_{t=0}^{T}$, where $\omega_{t}$ is conditionally Bernoulli distributed with probability $\left(B_{t}+C_{t}\right) / 2$. Let $n=5$. The first forecaster predicts at each time $t$ the interval $I_{t}^{1}$ which contains the 
TABLE 1

Monte Carlo power of detecting cross-calibration for different time periods $T$.

\begin{tabular}{lcccccc}
\hline \hline $\mathrm{T}$ & $10^{4}$ & $5 \cdot 10^{4}$ & $10^{5}$ & $5 \cdot 10^{5}$ & $10^{6}$ & $5 \cdot 10^{6}$ \\
\hline power & 0.112 & 0.254 & 0.333 & 0.699 & 0.847 & 0.994 \\
\hline
\end{tabular}

value $\left(B_{t}+C_{t}\right) / 2$, which is the probability that the realization $\omega_{t}$ is one. The second forecaster predicts the interval $I_{t}^{2}$ which contains the value $C_{t}$. Therefore, we expect that the first forecaster is cross-calibrated with respect to the second forecaster and should pass the test. The first forecaster passes the test if for all forecasting profiles $\ell=\left(\ell_{1}, \ell_{2}\right)$ where $\nu_{T}^{\ell}$ is positive $f_{T}^{\ell}$ lies in $\left[\left(\ell_{1}-1\right) / n, \ell_{1} / n\right]$. In Table 1 we see the result. For several $T$ we performed the test 1000 times. The second column of the table gives the Monte Carlo power of the test, that is, how often the test detected the cross-calibration of the first forecaster divided by 1000 . Table 1 shows that already for this rather simple example, the sample size $T$ needs to be large in order to come close to the theoretically predicted power of one.

\section{Diagnostic plots for Assessing Cross-calibration}

Complementary to the paper, we provide two further examples for diagnostic plots for marginal cross-calibration and for cross calibration.

Example 2.1. We consider the setting of Example 3.1 of the paper. That is, let $\mu$ be standard normally distributed, and, conditional on $\mu$, the outcome is $Y \sim \mathcal{N}(\mu, 1)$. Let $\tau$ take the values 1 or -1 with equal probability, independent of $Y$ and $\mu$. We consider the following four competing forecasters. The perfect forecaster $F_{1}$ predicts $\mathcal{N}(\mu, 1)$, the climatological forecaster is $F_{2}=\mathcal{N}(0,1)$, the unfocused forecaster $F_{3}$ gives the prediction $\frac{1}{2}\{\mathcal{N}(\mu, 1)+\mathcal{N}(\mu+\tau, 1)\}$, whereas the sign-reversed forecaster is $F_{4}=\mathcal{N}(-\mu, 1)$; see Table 1 of the paper for a summary.

As an example for checking cross-calibration empirically we note that all four forecaster are in the class of distribution functions $\mathcal{F}=\left\{F_{\lambda} \mid \lambda=(\mu, \sigma, \tau) \in\right.$ $\mathbb{R} \times(0, \infty) \times\{-1,0,1\}\}$ for $F_{\lambda}=\frac{1}{2}\{\mathcal{N}(\mu, \sigma)+\mathcal{N}(\mu+\tau, \sigma)\}$. In Figure 1 , we plotted the PIT-histograms of $Z_{2}$ and $Z_{3}$ conditional on the four bins $\mu \in I_{j}$ for $1 \leq j \leq 4$ with $I_{1}=(-\infty,-0.67), I_{2}=[-0.67,0), I_{3}=[0,0.67), I_{4}=[0.67, \infty)$ and $10^{\prime} 000$ simulations. The PIT-histograms confirm that $F_{3}$ is cross-calibrated with respect to $F_{1}, F_{2}, F_{4}$. On the other hand, $F_{2}$ is clearly not cross-calibrated with respect to any set of the other forecasters.

Example 2.2. In Example 2.5 of the paper, we have introduced the following setup. Let $\nu$ be uniformly distributed on $(5,20)$ and, conditionally on $\nu$, let $\sigma^{2}$ have an inverse chi-squared distribution with $\nu$ degrees of freedom. Conditional on $\nu$ and $\sigma$, the outcome $Y$ is normally distributed with mean zero and variance $\sigma^{2}$, and we consider two forecasters, a normally distributed forecaster $F_{1}=$ $\mathcal{N}\left(0, \sigma^{2}\right)$ and a t-distributed forecaster $F_{2}=t_{\nu}$. Theorem 2.9 implies that $F_{1}$ is marginally cross-calibrated with respect to itself and with respect to $F_{2}$.

imsart-ejs ver. 2014/10/16 file: cross-EJS-support.tex date: February 6, 2017 


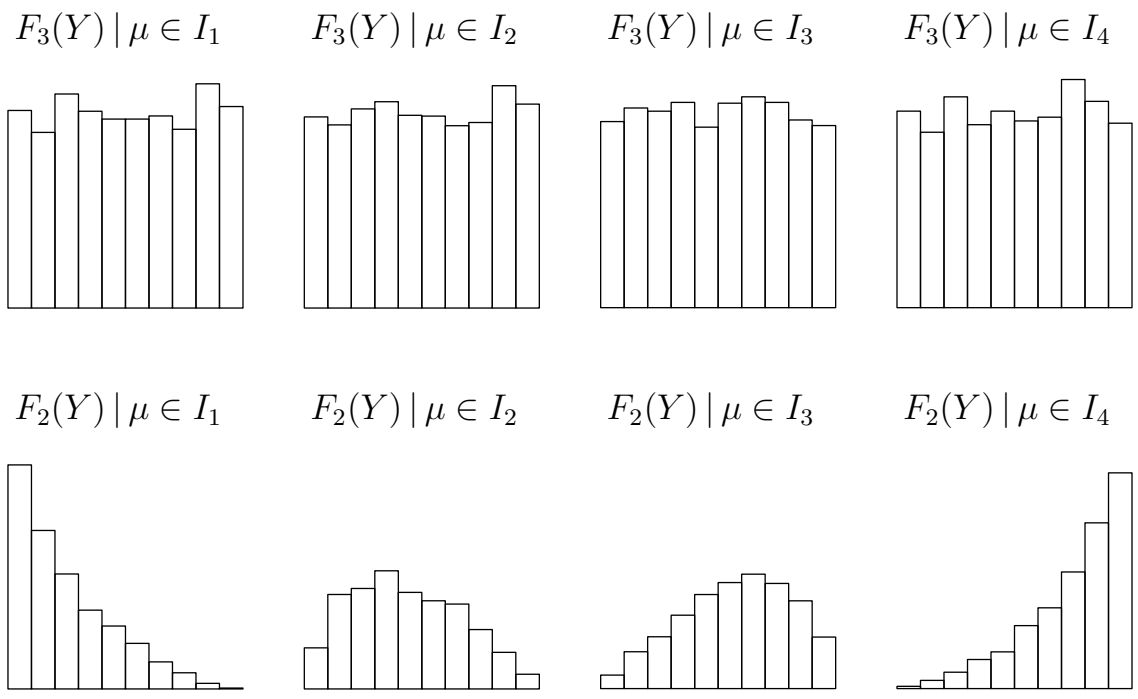

FIG 1. PIT-histogram plots for the forecasters $F_{3}$ and $F_{2}$ of Example 2.1 conditional on the mean in the top and bottom row, respectively. Here, $I_{1}=(-\infty,-0.67), I_{2}=[-0.67,0)$, $I_{3}=[0,0.67)$ and $I_{4}=[0.67, \infty)$ and 10'000 simulations have been performed.

Furthermore, $F_{2}$ is marginally cross-calibrated with respect to itself but $F_{2}$ is not marginally cross-calibrated with respect to $F_{1}$. Marginal cross-calibration plots for this scenario using 10'000 and 100'000 simulations are given in Figure 2. In this example, the lack of marginal cross-calibration can only be detected for an unrealistically large number of observations.

\section{The Score Regression Approach}

Held et al. (2010) suggest a significance test for ideal forecasters based on scoring rules. They use the continuous ranked probability score (CRPS) (Gneiting et al., 2005) and the Dawid-Sebastiani score (DSS) (Dawid and Sebastiani, 1999). Their approach relies on independent forecast-observation tuples, and this restriction remains, when generalizing their approach to a test for crossideal forecasters. Therefore, throughout this section we work in a one-period prediction space.

First, we recall some preliminaries on the CRPS and the DSS. Let $F$ and $f$ denote the predictive CDF of a forecaster and the predictive density function, respectively. Let $\mu$ and $\sigma^{2}$ be the predictive mean and variance, respectively. ${ }^{1}$

\footnotetext{
${ }^{1}$ In the prediction space setting, the quantities $F, f, \mu$, and $\sigma$ are random $\mathcal{A}_{0}$-measurable quantities. For ease of presentation, in this section we treat them as if they were deterministic, or, alternatively, one should consider all expectations and variances as conditional on the forecaster's information set $\mathcal{A}_{0} \subset \mathcal{A}$.
}

imsart-ejs ver. 2014/10/16 file: cross-EJS-support.tex date: February 6, 2017 


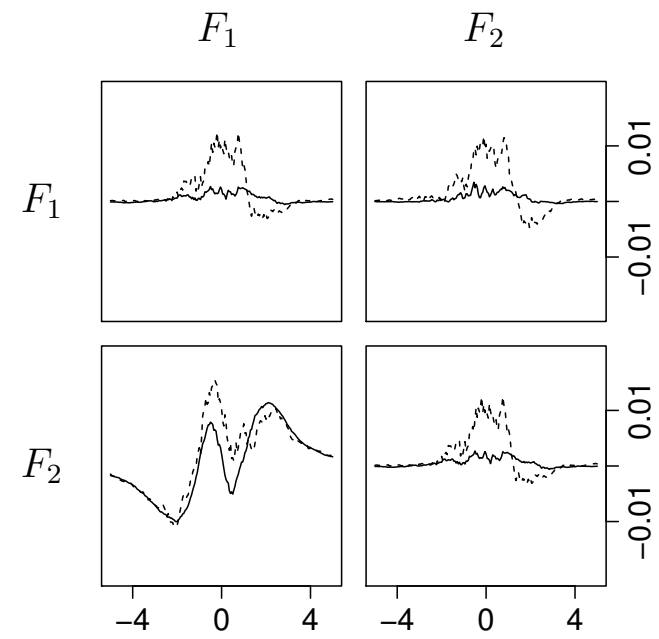

FIG 2. Figure 2: Marginal cross-calibration plots for the scenario in Example 2.2 of the paper for 10'000 simulation indicated by the dashed lines and 100'000 simulations indicated by the continuous lines. The $i$-th row and $j$-th column corresponds to the empirical version of Equation (4) of the paper in order to deduce whether $F_{i}$ is marginally cross-calibrated with respect to $F_{j}$ or not.

The observed value of $Y$ is denoted by $y$. The CRPS is given by

$$
\operatorname{CRPS}(F, y)=\int_{\infty}^{\infty}\{F(x)-\mathbb{1}(y \leq x)\}^{2} d x
$$

and the DSS by

$$
D S S(F, y)=\frac{1}{2}\left\{\log \left(\sigma^{2}\right)+\tilde{y}^{2}\right\},
$$

where $\tilde{y}=(y-\mu) / \sigma$. For a forecaster predicting a normal distribution $F=$ $\mathcal{N}\left(\mu, \sigma^{2}\right)$, the CRPS turns out to be

$$
\operatorname{CRPS}(F, y)=\sigma\left[\tilde{y}\{2 \Phi(\tilde{y})-1\}+2 \phi(\tilde{y})-\frac{1}{\sqrt{\pi}}\right]
$$

where $\Phi$ and $\phi$ are the CDF and the density of a standard normal distribution, respectively (Gneiting and Raftery, 2007). The CRPS is a strictly proper scoring rule relative to the class of probability measures with finite first moments; see Gneiting and Raftery (2007) for details on proper and strictly proper scoring rules.

For a normal prediction the DSS is the same as the classical logarithmic score $L S(f, y)=-\log \{f(y)\}$ up to a constant. The DSS is a proper scoring rule relative to the class of probability measures with finite second moment. It is strictly proper relative to any class of probability measures that are characterized by 
their first two moments, such as Gaussian measures or other location-scale families of distributions (Gneiting and Raftery, 2007).

We assume now that mean and variance of the predictive distribution $F$ match mean and variance of the outcome $Y$. The following properties of the CRPS and the DSS can be found in Held et al. (2010). For the DSS we get

$$
\mathbb{E}\{D S S(F, Y)\}=\frac{1}{2}+\log (\sigma) .
$$

If the distribution of $Y$ has finite fourth moment then $\operatorname{var}\{D S S(F, Y)\}$ is a constant that does not depend on $\mu$ or $\sigma$. If $Y$ has a normal distribution then $\operatorname{var}\{D S S(F, Y)\}=\frac{1}{2}$. Similar results for the CRPS are harder to obtain. Held et al. (2010) showed the following lemma.

Lemma 3.1. Let $X_{0}$ be a random variable with finite second moment. For $a \in \mathbb{R}$ and $b>0$, let $Y=a+b X_{0}$, let $F$ be the $C D F$ of $Y$, and $\sigma^{2}$ its variance. Then,

$$
\mathbb{E}\{C R P S(F, Y)\}=d \sigma \quad \text { and } \quad \operatorname{var}\{C R P S(F, y)\}=D \sigma^{2},
$$

where

$$
d=\frac{\mathbb{E}\left|X_{0}-X_{0}^{\prime}\right|}{2 \sqrt{\operatorname{var}\left(X_{0}\right)}} \quad \text { and } \quad D=\frac{\operatorname{var}\left\{\mathbb{E}\left(\left|X_{0}-X_{0}^{\prime}\right| \mid X_{0}\right)\right\}}{\operatorname{var}\left(X_{0}\right)}
$$

with $X_{0}^{\prime}$ an independent copy of $X_{0}$.

The lemma shows that for location-scale families of distributions the expected CRPS of an ideal forecast is proportional to the predictive standard deviation $\sigma$, and the variance of the CRPS is proportional to the predictive variance $\sigma^{2}$. For the family of normal distributions we have $d=1 / \sqrt{\pi}$ and $D=\{1 / 3-(4-$ $\sqrt{12}) / \pi\} \approx 0.16275$. The constants for other families can be calculated at least numerically.

For the score regression approach, we consider $N$ independent and identically distributed observations $\left(F_{1, n}, \ldots, F_{k, n}, Y_{n}, V_{n}\right), 1 \leq n \leq N$ in the one-period prediction space setting; see Definition 2.1 in the paper. The expectation of the DSS depends on the logarithm of the predictive standard deviation; see equation (1). Therefore, we stipulate a regression model of the form

$$
D S S\left(F_{1, n}, Y_{n}\right)=a+b_{1} \log \left(\sigma_{1, n}\right)+\ldots+b_{k} \log \left(\sigma_{k, n}\right)+\epsilon_{n},
$$

where $\sigma_{i, n}$ is the predictive standard deviation of $F_{i, n}$ and $\epsilon_{n}$ is an independent error with mean zero. Since the variance of the DSS is constant, irrespectively of the predictive variance, we can use a homoscedastic regression model to compute the least squares estimators $\hat{a}, \hat{b}_{1}, \ldots, \hat{b}_{k}$. In the case $k=1$ this is the same model as proposed at Held et al. (2010, eq. (7)). We need to assume that the scores have finite variance, which is fulfilled if $Y$ has a finite fourth moment (conditional on $\left.\mathcal{A}_{1}\right)$.

For the CRPS, motivated by (2), we stipulate the regression model

$$
\operatorname{CRPS}\left(F_{1, n}, Y_{n}\right)=c+d_{1} \sigma_{1, n}+\ldots+d_{k} \sigma_{k, n}+\epsilon_{n} .
$$

imsart-ejs ver. 2014/10/16 file: cross-EJS-support.tex date: February 6, 2017 
We have $\operatorname{var}\left\{\operatorname{CRPS}\left(F_{1, n}, Y_{n}\right)\right\} \propto \sigma_{1, n}$ and use a weighted regression analysis with weights $1 / \sigma_{1, n}$ to obtain estimators $\hat{c}, \hat{d}_{1}, \ldots, \hat{d}_{k}$; see for example Montgomery et al. (2001).

Both of these models can be used for testing if the forecaster $F_{1}$ is cross-ideal with respect to $\mathcal{A}_{1}=\sigma\left(F_{1}\right), \ldots, \mathcal{A}_{k}=\sigma\left(F_{k}\right)$ in case of a normal forecaster $F_{1}$. The DSS can also be used if the prediction is non-normal as emphasized in Held et al. (2010). The CRPS model is useful for any location-scale family of distributions.

We have the null hypotheses

$$
\begin{aligned}
& H_{0}: a=1 / 2, b_{1}=1 \text { and } b_{2}=\ldots=b_{k}=0 \text { for DSS } \\
& H_{0}: c=0, d_{1}=1 / \sqrt{\pi} \text { and } d_{2}=\ldots=d_{k}=0 \text { for CRPS }
\end{aligned}
$$

and perform a $\chi^{2}$-test. We use the test statistics

$$
\begin{aligned}
T_{D S S} & =\left(\hat{a}-1 / 2, \hat{b}_{1}-1, \hat{b}_{2}, \ldots, \hat{b}_{k}\right) \hat{\Sigma}_{D S S}^{-1}\left(\hat{a}-1 / 2, \hat{b}_{1}-1, \hat{b}_{2}, \ldots, \hat{b}_{k}\right)^{\top}, \\
T_{C R P S} & =\left(\hat{c}, \hat{d}_{1}-1 / \sqrt{\pi}, \hat{d}_{2}, \ldots, \hat{d}_{k}\right) \hat{\Sigma}_{C R P S}^{-1}\left(\hat{c}, \hat{d}_{1}-1 / \sqrt{\pi}, \hat{d}_{2}, \ldots, \hat{d}_{k}\right)^{\top},
\end{aligned}
$$

where $\hat{\Sigma}_{D S S}, \hat{\Sigma}_{C R P S}$ are the estimated covariance matrices. Both test statistics, $T_{D S S}$ and $T_{C R P S}$, are asymptotically $\chi^{2}$-distributed with $1+k$ degree of freedom and asymptotic $p$-values are given by

$$
\pi_{D S S}=1-\chi_{1+k}^{2}\left(T_{D S S}\right), \quad \text { and } \quad \pi_{C R P S}=1-\chi_{1+k}^{2}\left(T_{C R P S}\right) .
$$

If $k=1$, that is the case of just one forecaster, we obtain the test for an ideal forecaster suggested in Held et al. (2010). We call the tests presented in this section SRA tests as they are based on a score regression approach (SRA). As noted already by Held et al. (2010), SRA tests can only be used if each forecaster predicts at least two different variances, therefore we cannot apply it to Example 3.1 in the paper by Gneiting and Ranjan (2013). Instead, we consider the following setup for illustration.

Example 3.2. We consider two forecasters $F_{1, n}=\mathcal{N}\left(0,\left(1+\sigma_{n}\right)^{2}\right)$ and $F_{2, n}=$ $\mathcal{N}\left(0,\left(1+\sigma_{n}+\epsilon_{n}\right)^{2}\right)$, where $\sigma_{n} \sim \mathcal{U}([0,1])$ and $\epsilon_{n} \sim \mathcal{N}(0,1 / 16)$. The observations are $Y_{n} \sim \mathcal{N}\left(0,\left(1+\sigma_{n}\right)^{2}\right)$. It is clear, that $F_{1}$ is ideal with respect to $\mathcal{A}_{1}=\sigma\left(\sigma_{n}\right)$, $F_{2}$ is not ideal with respect to $\mathcal{A}_{2}=\sigma\left(\sigma_{n}, \epsilon_{n}\right), F_{1}$ is cross-ideal with respect to $F_{1}, F_{2}$, and $F_{2}$ is not cross-ideal with respect to $F_{1}, F_{2}$. In Table 3 , the Monte Carlo power of the CRPS test is displayed. The test is performed at significance level $\alpha=0.05$. The results are in accordance with the theoretical considerations. However, the Monte Carlo power of the test if $F_{2}$ is cross-ideal with respect to $F_{1}, F_{2}$ is higher than in the test if $F_{2}$ is ideal with respect to $F_{2}$. It is interesting to see that taking $F_{1}$ into account helps to detect that $F_{2}$ is not ideal with respect to $F_{2}$.

Example 3.3 (Example 2.2 continued). Considering the setup of Example 2.2, we used the DSS test to assess if the forecasters are cross-ideal. In Table 3 we present the Monte Carlo power of the test which is performed at significance 
TABLE 2

Monte Carlo power for the CRPS test

\begin{tabular}{lccccc}
\hline \hline$N$ & 30 & 50 & 100 & 200 & 500 \\
\hline$F_{1}$ wrt $F_{1}$ & 0.083 & 0.073 & 0.061 & 0.056 & 0.050 \\
$F_{1}$ wrt $F_{1}, F_{2}$ & 0.094 & 0.073 & 0.064 & 0.059 & 0.055 \\
$F_{2}$ wrt $F_{2}$ & 0.240 & 0.285 & 0.429 & 0.707 & 0.962 \\
$F_{2}$ wrt $F_{1}, F_{2}$ & 0.292 & 0.376 & 0.582 & 0.852 & 0.998 \\
\hline
\end{tabular}

Note: The test is performed at significance level $\alpha=0.05$ with sample size $N$ and $10^{\prime} 000$ simulations described in detail in Example 3.2.

TABLE 3

Monte Carlo power for the DSS test

\begin{tabular}{lccccc}
\hline \hline$N$ & 30 & 50 & 100 & 200 & 500 \\
\hline$F_{1}$ wrt $F_{1}$ & 0.085 & 0.075 & 0.064 & 0.055 & 0.056 \\
$F_{1}$ wrt $F_{1}, F_{2}$ & 0.084 & 0.072 & 0.065 & 0.057 & 0.054 \\
$F_{2}$ wrt $F_{2}$ & 0.111 & 0.096 & 0.090 & 0.074 & 0.072 \\
$F_{2}$ wrt $F_{1}, F_{2}$ & 0.286 & 0.416 & 0.718 & 0.963 & 1.000 \\
\hline
\end{tabular}

Note: The test is performed at significance level $\alpha=0.05$ with sample size $N$ and 10'000 simulations described in detail in Example 3.3.

level $\alpha=0.05$. The CRPS cannot be used for $F_{2}$ since the forecast is not normal. As expected, the test shows that $F_{1}$ is ideal with respect to $\mathcal{A}_{1}$ and also crossideal with respect to $\mathcal{A}_{1}, \mathcal{A}_{2}$. For a sample size of $N=200$ the level of the test is kept reasonably well. The forecaster $F_{2}$ is ideal with respect to $\mathcal{A}_{2}$ but fails to be cross-ideal with respect to $\mathcal{A}_{1}, \mathcal{A}_{2}$. The test shows a good power already for a sample size of $N=100$. However, for $F_{2}$ the test is slightly anticonservative even for a sample size of $N=500$.

Overall, we have presented three different approaches for testing cross-calibration, the CEP and LRA tests in Section 5 of the paper and the SRA tests. The first two approaches allow to test for cross-calibration of $F_{1}$ with respect to any subset $J \subset\{1, \ldots, k\}$, whereas the SRA tests only allow to test for $F_{1}$ being cross-ideal which is equivalent to requiring that $1 \in J$. The SRA tests, based on the score regression approach, require independent forecastobservation tuples, or, more precisely, independent sequences of realized score values $\operatorname{CRPS}\left(F_{1, n}, Y_{n}\right)$ or $\operatorname{DSS}\left(F_{1, n}, Y_{n}\right), 1 \leq n \leq N$, which may be a weaker requirement. They are asymptotic tests, that appear to be working well for sample sizes of at least $N=100$; see Tables 3 and 3. The SRA test with the CRPS works only for predictive distributions from one location-scale family, whereas the SRA test with the DSS requires only that the predictive distributions have finite fourth moments. In both cases, the predictive standard deviations have to differ for at least two observations. For the forecasters of Example 3.3, the SRA test with the DSS showed a better power than the CEP test, so it may be an interesting alternative despite the more restrictive assumptions. In particular, for sample size $N=200$ the SRA test had a power of 0.963 detecting that $F_{2}$ is not cross-ideal with respect to $F_{1}, F_{2}$, whereas the CEP test had a power of 0.464 . 


\section{Testing for Marginal Cross-calibration}

We consider two forecasters $F_{1}$ and $F_{2}$ within the prediction space setting. Our interest lies in

$$
S(y)=F_{2}(y)-\mathbb{1}\left\{F_{2}^{-1}\left(Z_{F_{1}}^{Y}\right) \leq y\right\}, \quad y \in \operatorname{supp}(Y),
$$

where $\operatorname{supp}(Y)$ denotes the support of of the observation $Y$. We would like to test if $\mathbb{E}_{\mathbb{Q}} S(y)=0$ for all $y \in \operatorname{supp}(Y)$, because this is equivalent to marginal cross-calibration of $F_{1}$ with respect to $F_{2}$; cf. Definition 2.7 of the paper.

We suppose that we have $N$ independent and identically distributed observations $\left(F_{1, n}, F_{2, n}, Y_{n}, V_{n}\right)$ for $1 \leq n \leq N$ in a prediction space and define for each $n$,

$$
S_{n}(y)=F_{2, n}(y)-\mathbb{1}\left\{F_{2, n}^{-1}\left(Z_{1, n}\right) \leq y\right\}, \quad y \in \operatorname{supp}(Y) .
$$

We pick a sequence $y_{0}<y_{1}<\ldots<y_{m}$ in the support of $Y$ and define

$$
\mathbf{S}_{\mathbf{n}}=\left(S_{n}\left(y_{0}\right), S_{n}\left(y_{1}\right), \ldots, S_{n}\left(y_{m}\right)\right)^{T},
$$

and $\overline{\mathbf{S}}_{\mathbf{N}}=(1 / N) \sum_{n=1}^{N} \mathbf{S}_{\mathbf{n}}$. Let $\Sigma_{N}=(1 / N) \sum_{n=1}^{N}\left(\mathbf{S}_{\mathbf{n}}-\overline{\mathbf{S}}_{\mathbf{N}}\right)\left(\mathbf{S}_{\mathbf{n}}-\overline{\mathbf{S}}_{\mathbf{N}}\right)^{T}$ be the sample covariance matrix. If $F_{1}$ is marginally cross-calibrated with respect to $F_{2}$, then $\mathbb{E}\left(\mathbf{S}_{\mathbf{n}}\right)=0$. Therefore, by standard arguments of probability theory, the test statistic $T=N \overline{\mathbf{S}}_{\mathbf{N}}^{T} \Sigma_{N}^{-1} \overline{\mathbf{S}}_{\mathbf{N}}$ converges in distribution to $\chi_{m}^{2}$, a chi-squared distribution with $m$ degrees of freedom.

We test the null hypothesis that $\mathbb{E}_{\mathbb{Q}} S(y)=0$ for all $y \in \operatorname{supp}(Y)$ for one particular finite distribution of $S(y)$. Therefore, the sequence $y_{1}, \ldots, y_{m}$ has to be chosen carefully. Simulations indicate that the level and power of the test is not much affected by the choice of $y_{1}, \ldots, y_{m}$. However, for small sample sizes the number of grid points $m$ should be rather small, otherwise the sample covariance matrix may be singular and can not be inverted to compute the test statistic. Another reason for singularity of the sample covariance matrix for small sample sizes may be the choice of a grid point $y$ such that the probability that $F_{i}^{-1}\left\{Z_{F_{j}}^{Y}\right\} \leq y$ is small. Unfortunately, for an individual test case, different choices of $y_{1}, \ldots, y_{m}$ may lead to completely different $p$-values, which makes the test useless in practice. We illustrate these effects in the following example.

Example 4.1. We consider the forecasters $F_{1}, \ldots, F_{4}$ and the observation $Y$ from Example 3.1 in the paper. Let $N=500$ be the number of observations from $\left(F_{i}, F_{j}, Y\right)$, for each pair $F_{i}$ and $F_{j}$ with $1 \leq i, j \leq 4$. The results in Table 4 show that the marginal cross-calibration test performs well overall, and the performance is relatively unaffected by the choice of different grid points $y_{1}, y_{2}, \ldots, y_{m}$. However, if we consider an increasing number of grid points for the same data set the $p$-value changes substantially. This is illustrated in Figure 3 for five different simulated data sets with $N=500$ and the null hypothesis that $F_{3}$ is marginally cross-calibrated with respect to $F_{4}$. 
TABLE 4

Monte Carlo power of the marginal cross-calibration test

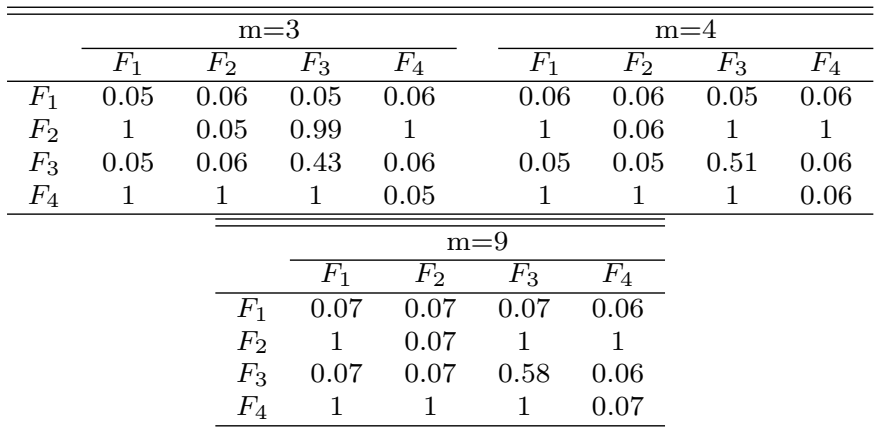

Note: The value in the $F_{i}$ row and $F_{j}$ column is the percentage of rejections of the null hypothesis that $F_{i}$ is marginally cross-calibrated with respect to $F_{j}$ at level $\alpha=0.05$ for the forecasters $F_{1}, F_{2}, F_{3}$ and $F_{4}$ in Example 4.1 for $10^{\prime} 000$ simulations. The test is performed for sample size $N=500$ and grid points

$$
\begin{aligned}
\left(y_{1}, y_{2}, y_{3}\right) & =(-0.95,0,0.95) \\
\left(y_{1}, y_{2}, y_{3}, y_{4}\right) & =(-1.19,-0.35,0.35,1.19), \\
\left(y_{1}, y_{2}, \ldots, y_{9}\right) & =(-1.81,-1.19,-0.74,-0.36,0.00,0.36,0.74,1.19,1.81),
\end{aligned}
$$

respectively, for $m=3,4,9$.

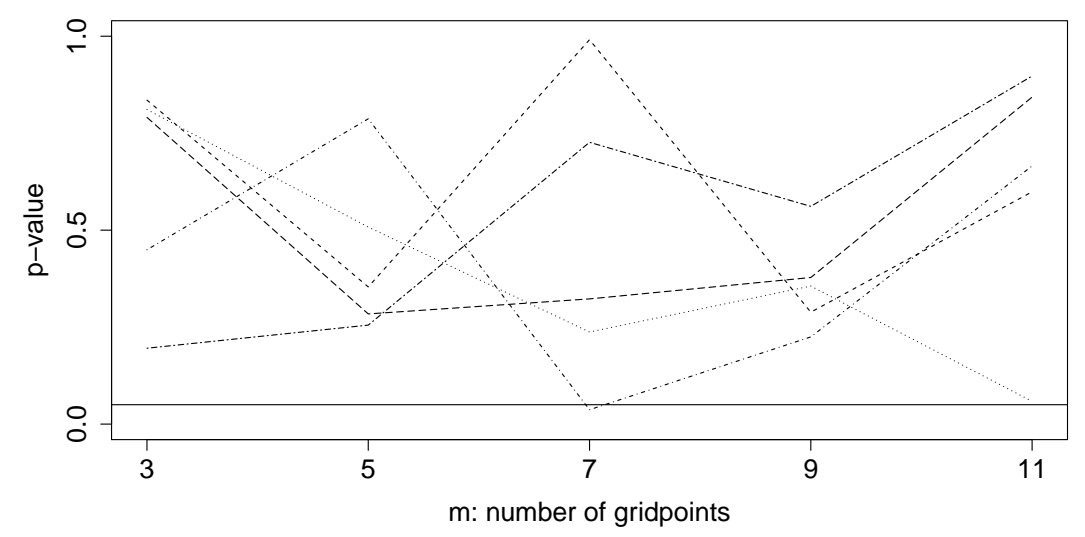

FIG 3. The p-values of the marginal cross-calibration test for five different simulated data sets and an increasing number of grid points. The horizontal line marks the $\alpha=0.05$ significance level. 


\section{References}

Dawid, A. P. and P. Sebastiani (1999). Coherent dispersion criteria for optimal experimental design. The Annals of Statistics 27, 65-81.

Feinberg, Y. and C. Stewart (2008). Testing multiple forecasters. Econometrica $76,561-582$.

Gneiting, T. and A. E. Raftery (2007). Strictly proper scoring rules, prediction, and estimation. Journal of the American Statistical Association 102, 359-378.

Gneiting, T., A. E. Raftery, A. H. Westveld, and T. Goldman (2005). Calibrated probabilistic forecasting using ensemble model output statistics and minimum CRPS estimation. Monthly Weather Review 133, 1098-1118.

Gneiting, T. and R. Ranjan (2013). Combining predictive distributions. Electronical Journal of Statistics 7, 1747-1782.

Held, L., K. Rufibach, and F. Balabdaoui (2010). A score regression approach to assess calibration of continuous probabilistic predictions. Biometrics 66, $1295-1305$.

Montgomery, D. C., E. A. Peck, and C. G. Vining (2001). Introduction to linear regression analysis (3rd ed.). John Wiley \& Sons, Inc.

R Core Team (2015). R: A Language and Environment for Statistical Computing. Vienna, Austria: R Foundation for Statistical Computing. ISBN 3-900051-07-0.

imsart-ejs ver. 2014/10/16 file: cross-EJS-support.tex date: February 6, 2017 